\title{
Seminormal Structure and Fixed Points of Cyclic Relatively Nonexpansive Mappings
}

\author{
Moosa Gabeleh ${ }^{1}$ and Naseer Shahzad ${ }^{2}$ \\ ${ }^{1}$ Department of Mathematics, Ayatollah Boroujerdi University, Boroujerd, Iran \\ ${ }^{2}$ Department of Mathematics, King Abdulaziz University, P.O. Box 80203, Jeddah 21589, Saudi Arabia
}

Correspondence should be addressed to Naseer Shahzad; nshahzad@kau.edu.sa

Received 14 September 2013; Accepted 10 December 2013; Published 16 January 2014

Academic Editor: Calogero Vetro

Copyright (C) 2014 M. Gabeleh and N. Shahzad. This is an open access article distributed under the Creative Commons Attribution License, which permits unrestricted use, distribution, and reproduction in any medium, provided the original work is properly cited.

Let $A$ and $B$ be two nonempty subsets of a Banach space $X$. A mapping $T: A \cup B \rightarrow A \cup B$ is said to be cyclic relatively nonexpansive if $T(A) \subseteq B$ and $T(B) \subseteq A$ and $\|T x-T y\| \leq\|x-y\|$ for all $(x, y) \in A \times B$. In this paper, we introduce a geometric notion of seminormal structure on a nonempty, bounded, closed, and convex pair of subsets of a Banach space $X$. It is shown that if $(A, B)$ is a nonempty, weakly compact, and convex pair and $(A, B)$ has seminormal structure, then a cyclic relatively nonexpansive mapping $T$ $: A \cup B \rightarrow A \cup B$ has a fixed point. We also discuss stability of fixed points by using the geometric notion of seminormal structure. In the last section, we discuss sufficient conditions which ensure the existence of best proximity points for cyclic contractive type mappings.

\section{Introduction}

Let $X$ be a Banach space and $C \subseteq X$. Recall that a mapping $T: C \rightarrow C$ is nonexpansive provided that $\|T x-T y\| \leq\|x-y\|$ for all $x, y \in C$. A closed convex subset $C$ of a Banach space $X$ has normal structure in the sense of Brodskii and Milman [1] if for each bounded, closed, and convex subset $D$ of $C$ which contains more than one point, there exists a point $x \in D$ which is not a diametral point; that is,

$$
\sup \{\|x-y\|: y \in D\}<\operatorname{diam}(D),
$$

where $\operatorname{diam}(D)$ is the diameter of $D$. The set $C$ is said to have fixed point property (FPP) if every nonexpansive mapping $T$ : $C \rightarrow C$ has a fixed point. In 1965, Kirk proved the following famous fixed theorem.

Theorem 1 (see [2]). Let $C$ be a nonempty, weakly compact, and convex subset of a Banach space X. If $C$ has normal structure, then $C$ has the FPP.

We mention that every compact and convex subset of a Banach space $X$ has normal structure (see [3]) and so has the FPP. Moreover, every bounded, closed, and convex subset of a uniformly convex Banach space $X$ has normal structure (see [4]) and then by Theorem 1 has the FPP. It is interesting to note that there exists a weakly compact and convex subset $C$ of $L^{1}[0,1]$ which does not have the fixed point property (see [5] for more information). In particular, $C$ cannot have normal structure.

In the current paper, we introduce a geometric notion of seminormal structure on a nonempty, closed, and convex pair of subsets of a Banach space $X$ and present a new fixed point theorem which is an extension of Kirk's fixed point theorem. We also study the stability of fixed points by using this geometric property. Finally, we establish a best proximity point theorem for a new class of mappings.

\section{Preliminaries}

In [6], Kirk et al. obtained an interesting extension of Banach contraction principle as follows.

Theorem 2 (see [6]). Let $A$ and $B$ be nonempty closed subsets of a complete metric space $(X, d)$. Suppose that $T: A \cup B \rightarrow$ $A \cup B$ is a cyclic mapping, that is, $T(A) \subseteq B$ and $T(B) \subseteq A$. If

$$
d(T x, T y) \leq \alpha d(x, y),
$$


for some $\alpha \in(0,1)$ and for all $x \in A, y \in B, T$ has a unique fixed point in $A \cap B$.

An interesting feature about the above observation is that continuity of $T$ is no longer needed. Indeed, simple examples can be constructed showing that discontinuous mappings can satisfy all the assumptions.

Let $A$ and $B$ be two nonempty subsets of a normed linear space $X$. A mapping $T: A \cup B \rightarrow A \cup B$ is said to be cyclic relatively nonexpansive if $T$ is cyclic and $\|T x-T y\| \leq$ $\|x-y\|$ whenever $x \in A$ and $y \in B$. It is clear that the class of cyclic relatively nonexpansive mappings contains the class of nonexpansive mappings as a subclass. Indeed, a cyclic relatively nonexpansive mapping need not to be continuous in general. Of course, if $A \cap B \neq \emptyset$, then the cyclic relatively nonexpansive mapping $T$ restricted to $A \cap B$ is nonexpansive. If $A \cap B=\emptyset$ then the fixed point equation $T x=x$ cannot have a solution; instead it is interesting to study the existence of best proximity points; that is, a point $p \in A \cup B$ such that

$$
\|p-T p\|=\operatorname{dist}(A, B):=\inf \{\|x-y\|: x \in A, y \in B\} .
$$

The relevance of best proximity points is that they provide optimal solutions for the problem of best approximation between two sets. Existence of best proximity points for cyclic relatively nonexpansive mappings was first studied in [7]. Afterwards, Eldred and Veeramani [8] studied the existence, uniqueness, and convergence of a best proximity point for cyclic contraction mappings in uniformly convex Banach spaces. For more information about the existence of best proximity points for various classes of cyclic mappings one can refer to $[5,9-22]$. For other related results, we refer to $[23,24]$.

To describe our results, we need some definitions and notations. We shall say that a pair $(A, B)$ of subsets of a Banach space $X$ satisfies a property if both $A$ and $B$ satisfy that property. For example, $(A, B)$ is convex if and only if both $A$ and $B$ are convex, $(A, B) \subseteq(C, D) \Leftrightarrow A \subseteq C$, and $B \subseteq D$. We will also adopt the notation

$$
\begin{gathered}
\delta_{x}(A)=\sup \{d(x, y): y \in A\} \quad \forall x \in X, \\
\delta(A, B)=\sup \left\{\delta_{x}(B): x \in A\right\}, \\
\operatorname{diam}(A)=\delta(A, A), \\
\Omega_{A}(B)=\inf \left\{\delta_{x}(B): x \in A\right\} \\
\Omega(A, B)=\max \left\{\Omega_{A}(B), \Omega_{B}(A)\right\}, \\
\mathscr{C}_{A}(B)=\left\{x \in A: \delta_{x}(B)=\Omega_{A}(B)\right\} .
\end{gathered}
$$

The closed and convex hull of a set $A$ will be denoted by $\overline{\operatorname{con}}(A)$. Given $(A, B)$ a pair of nonempty subsets of a Banach space, then its proximal pair is the pair $\left(A_{0}, B_{0}\right)$ given by

$$
\begin{aligned}
& A_{0}=\left\{x \in A:\left\|x-y^{\prime}\right\|=\operatorname{dist}(A, B) \text { for some } y^{\prime} \in B\right\}, \\
& B_{0}=\left\{y \in B:\left\|x^{\prime}-y\right\|=\operatorname{dist}(A, B) \text { for some } x^{\prime} \in A\right\} .
\end{aligned}
$$

Proximal pairs may be empty but, in particular, if $A$ and $B$ are nonempty weakly compact and convex then $\left(A_{0}, B_{0}\right)$ is a nonempty weakly compact convex pair in $X$.

Definition 3. A pair of sets $(A, B)$ is said to be proximal if $A=$ $A_{0}$ and $B=B_{0}$.

In [7], Eldred et al. introduced a geometric concept called proximal normal structure which generalizes the notion of normal structure introduced by Brodskii and Milman [1].

Definition 4. A convex pair $\left(K_{1}, K_{2}\right)$ in a Banach space $X$ is said to have proximal normal structure if for any bounded, closed, and convex proximal pair $\left(H_{1}, H_{2}\right) \subseteq\left(K_{1}, K_{2}\right)$ for which $\operatorname{dist}\left(H_{1}, H_{2}\right)=\operatorname{dist}\left(K_{1}, K_{2}\right)$ and $\delta\left(H_{1}, H_{2}\right)>$ $\operatorname{dist}\left(H_{1}, H_{2}\right)$, there exits $\left(x_{1}, x_{2}\right) \in H_{1} \times H_{2}$ such that

$$
\begin{aligned}
& \delta_{x_{1}}\left(H_{2}\right)<\delta\left(H_{1}, H_{2}\right), \\
& \delta_{x_{2}}\left(H_{1}\right)<\delta\left(H_{1}, H_{2}\right) .
\end{aligned}
$$

It was announced in [7] that every nonempty, bounded, closed, and convex pair of subsets of a uniformly convex Banach space $X$ has proximal normal structure (see Proposition 2.1 of [7]). We mention that a weaker notion than proximal normal structure which is called proximal quasinormal structure was introduced in [25] in order to study the existence of best proximity points for cyclic relatively Kannan nonexpansive mappings. [7].

The next best proximity point theorem was established in

Theorem 5 (see [7]). Let $(A, B)$ be a nonempty, weakly compact convex pair in a Banach space $X$, and suppose $(A, B)$ has proximal normal structure. Assume that $T: A \cup B \rightarrow A \cup B$ is a cyclic relatively nonexpansive mapping. Then $T$ has a best proximity point in both $A$ and $B$, that is, there exists $\left(x^{*}, y^{*}\right) \in$ $A \times B$ such that $\left\|x^{*}-T x^{*}\right\|=\left\|T y^{*}-y^{*}\right\|=\operatorname{dist}(A, B)$.

As a result of Theorem 5, the following corollary was obtained in [7].

Corollary 6. Let $(A, B)$ be a nonempty, bounded, closed, and convex pair in a uniformly convex Banach space $X$ and let $T$ : $A \cup B \rightarrow A \cup B$ be a cyclic relatively nonexpansive mapping. Then $T$ has a best proximity point.

\section{Seminormal Structure and a Fixed Point Theorem}

In this section motivated by Theorem 2 , we prove a fixed point theorem for cyclic relatively nonexpansive mappings which is an extension of Theorem 1 due to Kirk. We begin our main result with the following geometric notion.

Definition 7. A convex pair $(A, B)$ in a Banach space $X$ is said to have seminormal structure if for any bounded, closed and 
convex pair $\left(K_{1}, K_{2}\right) \subseteq(A, B)$ with $\delta\left(K_{1}, K_{2}\right)>0$, there exits $(p, q) \in K_{1} \times K_{2}$ such that

$$
\max \left\{\delta_{p}\left(K_{2}\right), \delta_{q}\left(K_{1}\right)\right\}<\delta\left(K_{1}, K_{2}\right)
$$

We note that the pair $(A, A)$ has seminormal structure if and only if $A$ has normal structure in the sense of Brodskii and Milman.

Here, we state the main result of this section.

Theorem 8. Let $(A, B)$ be a nonempty, weakly compact, and convex pair in a Banach space $X$, and suppose $(A, B)$ has seminormal structure. Assume that $T: A \cup B \rightarrow A \cup B$ is a cyclic relatively nonexpansive mapping. Then $T$ has a fixed point.

Proof. Let $\mathscr{F}$ denote the collection of all nonempty, closed, and convex pairs $(E, F) \subseteq(A, B)$ such that $T$ is cyclic on $E \cup F$. Since $(A, B)$ is a weakly compact and convex pair in $X$, the pair $\left(A_{0}, B_{0}\right)$ is also nonempty, closed, and convex pair in $X$. Moreover, $T$ is cyclic on $A_{0} \cup B_{0}$. Indeed, if $x \in A_{0}$ then there exists an element $y \in B_{0}$ such that $\|x-y\|=\operatorname{dist}(A, B)$. By the fact that $T$ is cyclic relatively nonexpansive, we have

$$
\|T x-T y\| \leq\|x-y\|=\operatorname{dist}(A, B),
$$

that is, $T x \in B_{0}$ and so, $T\left(A_{0}\right) \subseteq B_{0}$. Similarly, we can see that $T\left(B_{0}\right) \subseteq A_{0}$. Thus, $\left(A_{0}, B_{0}\right) \in \mathscr{F}$ and then $\mathscr{F}$ is nonempty. It follows from Zorn's lemma that $\mathscr{F}$ has a minimal element, say $\left(K_{1}, K_{2}\right)$. Since $T\left(K_{1}\right) \subseteq K_{2}$, we have $\overline{\operatorname{con}}\left(T\left(K_{1}\right)\right) \subseteq K_{2}$ and so,

$$
T\left(\overline{\operatorname{con}}\left(T\left(K_{1}\right)\right)\right) \subseteq T\left(K_{2}\right) \subseteq \overline{\operatorname{con}}\left(T\left(K_{2}\right)\right)
$$

Similarly, $T\left(\overline{\operatorname{con}}\left(T\left(K_{2}\right)\right)\right) \subseteq \overline{\operatorname{con}}\left(T\left(K_{1}\right)\right)$; that is, $T$ is cyclic on $\overline{\operatorname{con}}\left(T\left(K_{2}\right)\right) \cup \overline{\operatorname{con}}\left(T\left(K_{1}\right)\right)$. Minimality of $\left(K_{1}, K_{2}\right)$ concludes that

$$
\begin{aligned}
& \overline{\operatorname{con}}\left(T\left(K_{2}\right)\right)=K_{1}, \\
& \overline{\operatorname{con}}\left(T\left(K_{1}\right)\right)=K_{2} .
\end{aligned}
$$

Observe that if $\delta\left(K_{1}, K_{2}\right)=0$, then we must have $K_{1}=K_{2}=$ $\left\{x^{\star}\right\}$ for some $x^{\star} \in A \cup B$ and so, $x^{\star}$ is a fixed point of $T$ and we are finished. So, we may assume that $\delta\left(K_{1}, K_{2}\right)>0$. By seminormal structure there exist $(p, q) \in K_{1} \times K_{2}$ and $r \in(0,1)$ such that

$$
\max \left\{\delta_{p}\left(K_{2}\right), \delta_{q}\left(K_{1}\right)\right\} \leq r \delta\left(K_{1}, K_{2}\right)
$$

Put

$$
\begin{aligned}
& H_{1}:=\left\{u \in K_{1}: \delta_{u}\left(K_{2}\right) \leq r \delta\left(K_{1}, K_{2}\right)\right\}, \\
& H_{2}:=\left\{v \in K_{2}: \delta_{v}\left(K_{1}\right) \leq r \delta\left(K_{1}, K_{2}\right)\right\} .
\end{aligned}
$$

Note that $(p, q) \in H_{1} \times H_{2}$. We show that $\left(H_{1}, H_{2}\right)$ is a closed and convex pair in $X$. Let $\left\{u_{n}\right\}$ be a sequence in $H_{1}$ such that $u_{n} \rightarrow u$. Then for each $\varepsilon>0$ there exists $N \in \mathbb{N}$ such that $\left\|u_{n}-u\right\|<\varepsilon$ for all $n \geq N$. Since $K_{1}$ is closed, $u \in K_{1}$. Let $y \in K_{2}$. For all $n \geq N$ we have

$$
\begin{aligned}
\|u-y\| & \leq\left\|u-u_{n}\right\|+\left\|u_{n}-y\right\| \\
& \leq\left\|u-u_{n}\right\|+\delta_{u_{n}}\left(K_{2}\right) \\
& \leq\left\|u-u_{n}\right\|+r \delta\left(K_{1}, K_{2}\right) \\
& <\varepsilon+r \delta\left(K_{1}, K_{2}\right),
\end{aligned}
$$

which implies that $\delta_{u}\left(K_{2}\right) \leq r \delta\left(K_{1}, K_{2}\right)$. Hence, $u \in H_{1}$. Thereby, $H_{1}$ is closed. Similarly, we can see that $H_{2}$ is closed. Now, let $u_{1}, u_{2} \in H_{1}$ and $\lambda \in[0,1]$. For all $y \in K_{2}$ we have

$$
\begin{aligned}
\| \lambda u_{1} & +(1-\lambda) u_{2}-y \| \\
& \leq \lambda\left\|u_{1}-y\right\|+(1-\lambda)\left\|u_{2}-y\right\| \\
& \leq \lambda \delta_{u_{1}}\left(K_{2}\right)+(1-\lambda) \delta_{u_{2}}\left(K_{2}\right) \\
& \leq \lambda r \delta\left(K_{1}, K_{2}\right)+(1-\lambda) r \delta\left(K_{1}, K_{2}\right) \\
& =r \delta\left(K_{1}, K_{2}\right) .
\end{aligned}
$$

Therefore, $\delta_{\lambda u_{1}+(1-\lambda) u_{2}}\left(K_{2}\right) \leq r \delta\left(K_{1}, K_{2}\right)$ which deduces that $\lambda u_{1}+(1-\lambda) u_{2} \in H_{1}$. Hence, $H_{1}$ is convex. Similarly, $H_{2}$ is also convex. We assert that $T$ is cyclic on $H_{1} \cup H_{2}$. Suppose that $u \in H_{1}$. Let $x \in K_{1}$ and $\varepsilon>0$. Since $\operatorname{con}\left(T\left(K_{2}\right)\right)$ is dense in $K_{1}$, there exists $\sum_{i=1}^{j} \alpha_{i} T y_{i}$ such that $\sum_{i=1}^{j} \alpha_{i}=1,0 \leq \alpha_{i}$ and $y_{i} \in K_{2}$ for all $i=1,2, \ldots, j$ and $\left\|\sum_{i=1}^{j} \alpha_{i} T y_{i}-x\right\|<\varepsilon$. We have

$$
\begin{aligned}
\|T u-x\| & \leq\left\|T u-\sum_{i=1}^{j} \alpha_{i} T y_{i}\right\|+\left\|\sum_{i=1}^{j} \alpha_{i} T y_{i}-x\right\| \\
& <\left\|\sum_{i=1}^{j} \alpha_{i} T u-\sum_{i=1}^{j} \alpha_{i} T y_{i}\right\|+\varepsilon \leq \sum_{i=1}^{j} \alpha_{i}\left\|u-y_{i}\right\|+\varepsilon \\
& \leq \sum_{i=1}^{j} \alpha_{i} \delta_{u}\left(K_{2}\right)+\varepsilon \leq r \delta\left(K_{1}, K_{2}\right)+\varepsilon .
\end{aligned}
$$

This implies that $\delta_{T u}\left(K_{1}\right) \leq r \delta\left(K_{1}, K_{2}\right)$ and thus, Tu $\epsilon$ $H_{2}$. That is, $T\left(H_{1}\right) \subseteq H_{2}$. Similar argument concludes that $T\left(H_{2}\right) \subseteq H_{1}$ and so, $T$ is cyclic on $H_{1} \cup H_{2}$. Again, by the minimality of $\left(K_{1}, K_{2}\right)$ we obtain $H_{1}=K_{1}$ and $H_{2}=K_{2}$. Hence, for each $u \in K_{1}$ we have $\delta_{u}\left(K_{2}\right) \leq r \delta\left(K_{1}, K_{2}\right)$. Thus,

$$
\delta\left(K_{1}, K_{2}\right)=\sup _{u \in K_{1}} \delta_{u}\left(K_{2}\right) \leq r \delta\left(K_{1}, K_{2}\right),
$$

which is a contradiction.

In what follows, we give a sufficient condition which ensures that every nonempty, bounded, closed, and convex pair of subsets of a uniformly convex Banach space has seminormal structure.

Definition 9. A nonempty, bounded, closed, and convex pair $(A, B)$ of a normed linear space is said to have property $(D)$ 
provided that for each nonempty, closed, and convex pair $(E, F) \subseteq(A, B)$ we have

$$
\min \{\operatorname{diam}(E), \operatorname{diam}(F)\} \leq \delta(E, F) .
$$

Example 10. Let $(A, B)$ be a nonempty, bounded, closed, and convex pair in a normed linear space such that

$$
\min \{\operatorname{diam}(A), \operatorname{diam}(B)\} \leq \operatorname{dist}(A, B) .
$$

Then $(A, B)$ has the $(D)$ property.

Proof. Suppose that $(E, F) \subseteq(A, B)$ is a nonempty, closed, and convex pair. Then we have

$$
\begin{aligned}
\min & \{\operatorname{diam}(E), \operatorname{diam}(F)\} \\
& \leq \min \{\operatorname{diam}(A), \operatorname{diam}(B)\} \\
& \leq \operatorname{dist}(A, B) \leq \operatorname{dist}(E, F) \leq \delta(E, F) ;
\end{aligned}
$$

that is, $(A, B)$ has the property $(D)$.

Let $X$ be a uniformly convex Banach space with modulus of convexity $\delta$. Then $\delta(\varepsilon)>0$ for $\varepsilon>0$. Moreover, if $x, y, z \in$ $X, R>0$, and $r \in[0,2 R]$ we have

$$
\left\{\begin{array}{l}
\|x-z\| \leq R \\
\|y-z\| \leq R \\
\|x-y\| \geq r
\end{array} \Longrightarrow\left\|\frac{x+y}{2}-z\right\| \leq\left(1-\delta\left(\frac{r}{R}\right)\right) R .\right.
$$

Motivated by the fact that every nonempty, bounded, closed, and convex subset of a uniformly convex Banach space $X$ has normal structure, we establish the following result concerning seminormal structure in uniformly convex Banach spaces.

Proposition 11. Let $(A, B)$ be a nonempty, bounded, closed, and convex pair in a uniformly convex Banach space $X$ such that $(A, B)$ has the property $(D)$. Then $(A, B)$ has seminormal structure.

Proof. Let $\left(K_{1}, K_{2}\right) \subseteq(A, B)$ be a nonempty, closed, and convex pair. Put, $R:=\delta\left(K_{1}, K_{2}\right)$ and $r:=$ $\min \left\{\operatorname{diam}\left(K_{1}\right), \operatorname{diam}\left(K_{2}\right)\right\}$. Since $(A, B)$ has the property $(D)$, we have $r \leq R$. There exists $x_{1}, x_{2} \in K_{1}$ such that $\left\|x_{1}-x_{2}\right\| \geq$ $(1 / 2) \operatorname{diam}\left(K_{1}\right)$. Now, for each $y \in K_{2}$ we have

$$
\begin{gathered}
\left\|x_{1}-y\right\| \leq R, \\
\left\|x_{2}-y\right\| \leq R, \\
\left\|x_{1}-x_{2}\right\| \geq \frac{1}{2} r .
\end{gathered}
$$

It follows from the uniformly convexity of the Banach space $X$ that

$$
\left\|y-\frac{x_{1}+x_{2}}{2}\right\| \leq\left(1-\delta\left(\frac{(1 / 2) r}{R}\right)\right) R .
$$

If we set $c:=\delta((1 / 2) r / R)$ and $p:=\left(x_{1}+x_{2}\right) / 2 \in K_{1}$, then for each $y \in K_{2}$ we have $\|y-p\| \leq c R$ which concludes that $\delta_{p}\left(K_{2}\right) \leq c R<R$. Similar argument implies that there exists an element $q \in K_{2}$ such that $\delta_{q}\left(K_{1}\right)<R$, which completes the proof.

The following corollary obtains from Theorem 8 and Proposition 11, immediately.

Corollary 12. Let $(A, B)$ be a nonempty, bounded, closed, and convex pair in a uniformly convex Banach space $X$ such that $(A, B)$ has the property $(D)$. Assume that $T: A \cup B \rightarrow A \cup B$ is a cyclic relatively nonexpansive mapping. Then $T$ has a fixed point.

It is interesting to note that by admitting property $(D)$ to the assumptions of Corollary 6, we conclude the existence of fixed points for cyclic relatively nonexpansive mappings instead of the existence of best proximity points. Here, we raise the following question.

Question 1. It is interesting to ask whether one considers a better condition than the property $(D)$ in Corollary 12 such that the cyclic relatively nonexpansive mapping $T$ has a fixed point.

Example 13. Let us consider $X=\mathbb{R}$ with the usual metric. Let $A:=[-1,0]$ and $B:=[0,1]$. Define $T: A \cup B \rightarrow A \cup B$ by

$$
T(x)=\left\{\begin{aligned}
x+1 & \text { if } x \in A \cap\left[-1,-\frac{1}{2}\right] \\
-\frac{x}{2} & \text { if } x \in A \cap\left(-\frac{1}{2}, 0\right] \\
-x & \text { if } x \in B .
\end{aligned}\right.
$$

We claim that $T$ is cyclic relatively nonexpansive. In this order, we consider the following two cases.

Case 1. If $x \in A \cap[-1,-1 / 2]$ and $y \in B$, then

$$
|T x-T y|=x+1+y \leq y-x=|x-y| \text {. }
$$

Case 2. If $x \in A \cap(-1 / 2,0]$ and $y \in B$, then

$$
|T x-T y|=y-\frac{x}{2} \leq y-x=|x-y| \text {. }
$$

Thus, $T$ is a cyclic relatively nonexpansive mapping. Corollary 12 guarantees the existence of fixed point for the mapping $T$ which is a point $x^{\star}=0$. It is interesting to note that the existence of a fixed point for the mapping $T$ cannot be obtained from Theorem 1 due to Kirk because $T$ is not continuous.

In 1974, Lim proved the following common fixed point theorem.

Theorem 11 (see [26]). Let $K$ be a nonempty, weakly compact, and convex subset of a Banach space $X$. If $K$ has normal structure, then any family of commuting nonexpansive mappings on $K$ into itself admits a common fixed point. 
The following result is a generalized version of Lim's theorem.

Theorem 12. Let $(A, B)$ be a nonempty, weakly compact and convex pair of subsets of a Banach space $X$ such that $(A, B)$ has seminormal structure. Suppose that $\mathfrak{F}=\left\{T_{\alpha}: \alpha \in I\right\}$ is a family of commuting cyclic relatively nonexpansive mappings on $A \cup B$ with some index set $I$. Then $\mathfrak{F}$ has a common fixed point, that is, there exists an element $x^{\star} \in A \cap B$ such that

$$
x^{\star}=T_{\alpha} x^{\star}, \quad \forall \alpha \in I .
$$

At the end of this section, we consider cyclic mappings which do not increase large distances. Our purpose is not to seek fixed points but rather to determine what can be said about minimal displacement for such cyclic mappings.

Definition 13. Let $(A, B)$ be a nonempty pair of subsets of a normed linear space. A mapping $T: A \cup B \rightarrow A \cup B$ is said to be cyclic relatively $h$-nonexpansive for $h>0$ if

$$
\|T x-T y\| \leq \max \{\|x-y\|, h\}, \quad \forall(x, y) \in A \times B .
$$

Our result is the following. The surprising aspect of the conclusion of the following theorem is the fact that cyclic relatively $h$-nonexpansive mappings have minimal displacement strictly less than $h$ in the presence of seminormality.

Theorem 14. Let $(A, B)$ be a nonempty, weakly compact, and convex pair in a Banach space $X$ such that $(A, B)$ has seminormal structure. Let $T: A \cup B \rightarrow A \cup B$ be a cyclic relatively $h$-nonexpansive mapping for $h>0$. Then there exists an element $x^{\star} \in A \cup B$ such that

$$
\left\|x^{\star}-T x^{\star}\right\| \leq h
$$

Proof. Proceeding in a similar way as in Theorem 8, we obtain, by minimality, that $K_{1}=\overline{\operatorname{con}}\left(T\left(K_{2}\right)\right)$ and $K_{2}=$ $\overline{\operatorname{con}}\left(T\left(K_{1}\right)\right)$. If $\delta\left(K_{1}, K_{2}\right)=0$, then there is nothing to prove. So, assume that $\delta\left(K_{1}, K_{2}\right)>0$. Since $(A, B)$ has seminormal structure, there exist $r \in(0,1)$ and $(u, v) \in K_{1} \times K_{2}$ such that

$$
\max \left\{\delta_{u}\left(K_{2}\right), \delta_{v}\left(K_{1}\right)\right\} \leq r \delta\left(K_{1}, K_{2}\right) .
$$

By the fact that $\left(K_{1}, K_{2}\right)$ is a weakly compact and convex pair in $X$, the pair $\left(\mathscr{C}_{K_{1}}\left(K_{2}\right), \mathscr{C}_{K_{2}}\left(K_{1}\right)\right) \subseteq\left(K_{1}, K_{2}\right)$ is nonempty, closed, and convex. Let $\left(z_{1}, z_{2}\right) \in \mathscr{C}_{K_{1}}\left(K_{2}\right) \times \mathscr{C}_{K_{2}}\left(K_{1}\right)$. Then $\delta_{z_{1}}\left(K_{2}\right)=\Omega_{K_{1}}\left(K_{2}\right)$ and $\delta_{z_{2}}\left(K_{1}\right)=\Omega_{K_{2}}\left(K_{1}\right)$. If $\Omega\left(K_{1}, K_{2}\right)<h$, thus

$$
\left\|z_{1}-T z_{1}\right\| \leq \delta_{z_{1}}\left(K_{2}\right)=\Omega_{K_{1}}\left(K_{2}\right) \leq \Omega\left(K_{1}, K_{2}\right)<h,
$$

and again we are finished. So, we assume that $h \leq \Omega\left(K_{1}, K_{2}\right)$. Let $y \in K_{2}$. If $\left\|z_{1}-y\right\| \geq h$, then

$$
\begin{aligned}
\left\|T z_{1}-T y\right\| & \leq \max \left\{\left\|z_{1}-y\right\|, h\right\}=\left\|z_{1}-y\right\| \\
& \leq \delta_{z_{1}}\left(K_{2}\right)=\Omega_{K_{1}}\left(K_{2}\right) \leq \Omega\left(K_{1}, K_{2}\right) .
\end{aligned}
$$

On the other hand, if $\left\|z_{1}-y\right\|<h$, then

$$
\left\|T z_{1}-T y\right\| \leq \max \left\{\left\|z_{1}-y\right\|, h\right\}=h \leq \Omega\left(K_{1}, K_{2}\right) .
$$

Therefore, in either case $T y \in \mathscr{B}\left(T z_{1} ; \Omega\left(K_{1}, K_{2}\right)\right)$. Hence, $K_{1}=\overline{\operatorname{con}}\left(T\left(K_{2}\right)\right) \subseteq \mathscr{B}\left(T z_{1}, \Omega\left(K_{1}, K_{2}\right)\right)$ which concludes that $\delta_{T z_{1}}\left(K_{1}\right) \leq \Omega_{K_{2}}\left(K_{1}\right)$. That is, $T z_{1} \in \mathscr{C}_{K_{2}}\left(K_{1}\right)$. Similarly, we can see that $T z_{2} \in \mathscr{C}_{K_{1}}\left(K_{2}\right)$. Thereby, $T$ is cyclic on $\mathscr{C}_{K_{1}}\left(K_{2}\right) \cup \mathscr{C}_{K_{2}}\left(K_{1}\right)$. It follows from the minimality of $\left(K_{1}, K_{2}\right)$ that $\mathscr{C}_{K_{1}}\left(K_{2}\right)=K_{1}$ and $\mathscr{C}_{K_{2}}\left(K_{1}\right)=K_{2}$. Now, for each $x \in K_{1}$ we have $\delta_{x}\left(K_{2}\right)=\Omega_{K_{1}}\left(K_{2}\right)$ and so,

$$
\delta\left(K_{1}, K_{2}\right)=\sup _{x \in K_{1}} \delta_{x}\left(K_{2}\right)=\Omega_{K_{1}}\left(K_{2}\right)=\delta_{u}\left(K_{2}\right) .
$$

Also, we can see that $\delta\left(K_{1}, K_{2}\right)=\delta_{v}\left(K_{1}\right)$. Hence,

$$
\delta\left(K_{1}, K_{2}\right)=\max \left\{\delta_{u}\left(K_{2}\right), \delta_{v}\left(K_{1}\right)\right\} \leq r \delta\left(K_{1}, K_{2}\right),
$$

which is a contradiction.

\section{A Best Proximity Point Theorem}

Recently, Kosuru and Veeramani introduced a concept of pointwise cyclic contractions as follows.

Definition 15 (see [27]). Let $(A, B)$ be a pair of subsets of a metric space $(X, d)$. A mapping $T: A \cup B \rightarrow A \cup B$ is said to be a pointwise cyclic contraction if $T$ is cyclic and for each $(x, y) \in A \times B$ there exist $0 \leq \alpha(x)<1,0 \leq \alpha(y)<1$ such that

$$
\begin{aligned}
d(T x, T y) \leq & \alpha(x) d(x, y) \\
& +(1-\alpha(x)) \operatorname{dist}(A, B) \quad \forall y \in B, \\
d(T x, T y) \leq & \alpha(y) d(x, y) \\
& +(1-\alpha(y)) \operatorname{dist}(A, B) \quad \forall x \in A .
\end{aligned}
$$

The following best proximity point theorem was proved in [27] by using a geometric notion of proximal normal structure.

Theorem 16 (Theorem 4.1 of [27]). Let $(A, B)$ be a nonempty, weakly compact, and convex pair of a Banach space $X$. If $T$ : $A \cup B \rightarrow A \cup B$ is a pointwise cyclic contraction mapping, then $T$ has a best proximity point.

To establish our results, we introduce the following class of cyclic mappings.

Definition 17. Let $(A, B)$ be a pair of subsets of a metric space $(X, d)$. A mapping $T: A \cup B \rightarrow A \cup B$ is said to be a generalized pointwise cyclic contraction if $T$ is cyclic and for each $(x, y) \in A \times B$ there exist $0 \leq \alpha(x), \beta(x)<1,0 \leq \alpha(y)$, and $\beta(y)<1$ such that $\alpha(x)+\beta(x)<1, \alpha(y)+\beta(y)<1$ and

$$
\begin{aligned}
d(T x, T y) \leq & \alpha(x) d(x, y)+\beta(x) m(x, y) \\
& +(1-(\alpha(x)+\beta(x))) \operatorname{dist}(A, B) \quad \forall y \in B, \\
d(T x, T y) \leq & \alpha(y) d(x, y)+\beta(y) m(x, y) \\
& +(1-(\alpha(y)+\beta(y))) \operatorname{dist}(A, B) \quad \forall x \in A,
\end{aligned}
$$


where $m(x, y):=\min \{d(x, T x), d(y, T y)\}$ for all $(x, y) \in A \times$ $B$.

Obviously, every generalized pointwise cyclic contraction is a cyclic relatively nonexpansive mapping. Also, the class of generalized pointwise cyclic contractions contains the class of pointwise cyclic contractions as a subclass. The next example shows that the reverse implication does not hold.

Example 18. Let $X:=\mathbb{R}$ with the usual metric. For $A=B=$ $[0,1 / 4]$, define $T: A \cup B \rightarrow A \cup B$ with

$$
T x= \begin{cases}\frac{1}{16} x & \text { if } 0 \leq x \leq \frac{1}{8}, \\ 0 & \text { if } \frac{1}{8}<x \leq \frac{1}{4} .\end{cases}
$$

Then $T$ is generalized pointwise cyclic contraction. Indeed, if either $0 \leq x, y \leq 1 / 8$ or $1 / 8<x, y \leq 1 / 4$ then the result follows, easily. Also, if $0 \leq x \leq 1 / 8$ and $1 / 8<y \leq 1 / 4$, then for every $x, y \in A$ if $\alpha(x)=\beta(x)=7 / 16$, we have

$$
\begin{aligned}
d(T x, T y) & =\frac{1}{16} x \leq \frac{7}{16}(y-x)+\frac{102}{256} x \\
& =\alpha(x) d(x, y)+\beta(x) m(x, y) .
\end{aligned}
$$

which implies that $T$ is generalized pointwise cyclic contraction. It is interesting to note that $T$ is not pointwise cyclic contraction. In fact, if $x=1 / 8$ and $y=13 / 100$ and if $T$ is pointwise cyclic contraction then we must have

$$
\begin{aligned}
d(T x, T y) & =\frac{1}{16} \times \frac{1}{8} \leq \alpha(x) d(x, y) \\
& =\alpha\left(\frac{1}{8}\right)\left(\frac{13}{100}-\frac{1}{8}\right),
\end{aligned}
$$

which deduces that $200 / 128 \leq \alpha(1 / 8)$ which is a contradiction. Besides, we mention that $T$ is not nonexpansive because $T$ is not continuous.

Here, we state the main result of this section.

Theorem 19. Let $(A, B)$ be a nonempty, weakly compact, and convex pair of a Banach space X. If $T: A \cup B \rightarrow A \cup B$ is a generalized pointwise cyclic contraction mapping, then $T$ has a best proximity point.

Proof. Since $T$ is cyclic relatively nonexpansive, we can apply Theorem 8 to deduce the existence of a minimal weakly compact convex pair $\left(K_{1}, K_{2}\right)$ such that $\overline{c o}\left(T\left(K_{2}\right)\right)=K_{1}$ and $\overline{\mathrm{co}}\left(T\left(K_{1}\right)\right)=K_{2}$. Let $x \in K_{1}$ be fixed. For each $z \in K_{2}$ we have

$$
\begin{aligned}
\|T x-T z\| \leq & \alpha(x) d(x, z)+\beta(x) m(x, z) \\
& +(1-(\alpha(x)+\beta(x))) \operatorname{dist}(A, B) \\
\leq & \alpha(x) \delta_{x}\left(K_{2}\right)+\beta(x) \delta_{x}\left(K_{2}\right) \\
& +(1-(\alpha(x)+\beta(x))) \operatorname{dist}(A, B) \\
= & (\alpha(x)+\beta(x)) \delta_{x}\left(K_{2}\right) \\
& +(1-(\alpha(x)+\beta(x))) \operatorname{dist}(A, B) .
\end{aligned}
$$

Hence, for all $z \in K_{2}$, we have $T z \in \mathscr{B}(T x ;(\alpha(x)+$ $\left.\beta(x)) \delta_{x}\left(K_{2}\right)+(1-(\alpha(x)+\beta(x))) \operatorname{dist}(A, B)\right)$, and then

$$
\begin{aligned}
T\left(K_{2}\right) \subseteq \mathscr{B}(T x & ;(\alpha(x)+\beta(x)) \delta_{x}\left(K_{2}\right) \\
+ & (1-(\alpha(x)+\beta(x))) \operatorname{dist}(A, B)) .
\end{aligned}
$$

Therefore,

$$
\begin{aligned}
K_{1}= & \overline{\operatorname{co}}\left(T\left(K_{2}\right)\right) \subseteq \mathscr{B}\left(T x ;(\alpha(x)+\beta(x)) \delta_{x}\left(K_{2}\right)\right. \\
& +(1-(\alpha(x)+\beta(x))) \operatorname{dist}(A, B)),
\end{aligned}
$$

which concludes that

$$
\begin{aligned}
\delta_{T x}\left(K_{1}\right) \leq & (\alpha(x)+\beta(x)) \delta_{x}\left(K_{2}\right) \\
& +(1-(\alpha(x)+\beta(x))) \operatorname{dist}(A, B), \quad \forall x \in K_{1} .
\end{aligned}
$$

Similar argument implies that

$$
\begin{aligned}
\delta_{T y}\left(K_{2}\right) \leq & (\alpha(y)+\beta(y)) \delta_{y}\left(K_{1}\right) \\
& +(1-(\alpha(y)+\beta(y))) \operatorname{dist}(A, B), \quad \forall y \in K_{2} .
\end{aligned}
$$

Now, let $(u, v)$ be an arbitrary element in $K_{1} \times K_{2}$. We may suppose that $\delta_{u}\left(K_{2}\right) \leq \delta_{v}\left(K_{1}\right)$. Put $r:=\delta_{u}\left(K_{2}\right)$. Let

$$
\begin{aligned}
& L_{1}:=\left\{x \in K_{1}: \delta_{x}\left(K_{2}\right) \leq r\right\}, \\
& L_{2}:=\left\{y \in K_{2}: \delta_{x}\left(K_{1}\right) \leq r\right\} .
\end{aligned}
$$

We note that $u \in L_{1}$ and by the fact that

$$
\begin{aligned}
\delta_{T u}\left(K_{1}\right) \leq & (\alpha(x)+\beta(x)) \delta_{u}\left(K_{2}\right) \\
& +(1-(\alpha(x)+\beta(x))) \operatorname{dist}(A, B) \\
\leq & \delta_{u}\left(K_{2}\right)=r,
\end{aligned}
$$

we have $T u \in L_{2}$. Moreover, it is easy to see that

$$
\begin{aligned}
& L_{1}=\left[\bigcap_{y \in K_{2}} \mathscr{B}(y ; r)\right] \cap K_{1}, \\
& L_{2}=\left[\bigcap_{x \in K_{1}} \mathscr{B}(x ; r)\right] \cap K_{2} ;
\end{aligned}
$$

that is, $\left(L_{1}, L_{2}\right) \subseteq\left(K_{1}, K_{2}\right)$ is a nonempty, closed, and convex pair in $X$. Besides, if $x \in L_{1}$ then we have

$$
\begin{aligned}
\delta_{T x}\left(K_{1}\right) \leq & (\alpha(x)+\beta(x)) \delta_{x}\left(K_{2}\right) \\
& +(1-(\alpha(x)+\beta(x))) \operatorname{dist}(A, B) \\
\leq & \delta_{x}\left(K_{2}\right) \leq r,
\end{aligned}
$$

which implies that $T x \in L_{2}$ and so, $T\left(L_{1}\right) \subseteq L_{2}$. Similarly, $T\left(L_{2}\right) \subseteq L_{1}$. Therefore, $T$ is cyclic on $L_{1} \cup L_{2}$. Now, by 
the minimality of $\left(K_{1}, K_{2}\right)$ we must have $L_{1}=K_{1}$ and $L_{2}=$ $K_{2}$. Thereby,

$$
\delta_{x}\left(K_{2}\right) \leq r=\delta_{u}\left(K_{2}\right), \quad \forall x \in K_{1},
$$

which implies that

$$
\begin{aligned}
\delta\left(K_{1}, K_{2}\right) & =\sup _{x \in K_{1}} \delta_{x}\left(K_{2}\right) \leq \delta_{u}\left(K_{2}\right) \\
& \leq \delta_{v}\left(K_{1}\right) \leq \delta\left(K_{1}, K_{2}\right) .
\end{aligned}
$$

Hence, for all $(u, v) \in K_{1} \times K_{2}$ we have

$$
\delta_{u}\left(K_{2}\right)=\delta_{v}\left(K_{1}\right)
$$

If $\delta_{u}\left(K_{2}\right)=\operatorname{dist}(A, B)$, then $u$ is a best proximity point of $T$ and we are finished. So, let $\delta_{u}\left(K_{2}\right)>\operatorname{dist}(A, B)$. We have

$$
\begin{aligned}
\delta_{u}\left(K_{2}\right)= & \delta_{T u}\left(K_{1}\right) \leq(\alpha(u)+\beta(u)) \delta_{u}\left(K_{2}\right) \\
& +(1-(\alpha(u)+\beta(u))) \operatorname{dist}(A, B) \\
< & \delta_{u}\left(K_{2}\right),
\end{aligned}
$$

which is a contradiction. Therefore, for all $(u, v) \in K_{1} \times K_{2}$ we must have

$$
\|u-T u\|=\|T v-v\|=\operatorname{dist}(A, B) .
$$

Remark 20. Note that Theorem 19 was proved directly and without using the notion of proximal normal structure.

Remark 21. Theorem 19 holds once the minimal sets $K_{1}$ and $K_{2}$ have been fixed and the cyclic mapping $T: A \cup B \rightarrow$ $A \cup B$ satisfies the condition that for each $(x, y) \in A \times B$ there exist $0 \leq \alpha(x), \beta(x)<1,0 \leq \alpha(y)$, and $\beta(y)<1$ such that $\alpha(x)+\beta(x)<1, \alpha(y)+\beta(y)<1$ and

$$
\begin{aligned}
d(T x, T y) \leq & \alpha(x) d(x, y)+\beta(x) M(x, y) \\
& +(1-(\alpha(x)+\beta(x))) \operatorname{dist}(A, B), \quad \forall y \in B, \\
d(T x, T y) \leq & \alpha(y) d(x, y)+\beta(y) M(x, y) \\
& +(1-(\alpha(y)+\beta(y))) \operatorname{dist}(A, B), \quad \forall x \in A,
\end{aligned}
$$

where $M(x, y):=\min \left\{\delta_{x}\left(K_{2}\right), \delta_{y}\left(K_{1}\right)\right\}$ for all $(x, y) \in A \times B$.

\section{Conflict of Interests}

The authors declare that there is no conflict of interests regarding the publication of this paper.

\section{Acknowledgments}

This paper was funded by the Deanship of Scientific Research (DSR), King Abdulaziz University, Jeddah. Naseer Shahzad acknowledges with thanks DSR for financial support.

\section{References}

[1] M. S. Brodskii and D. P. Milman, "On the center of a convex set," Doklady Akademii Nauk SSSR, vol. 59, pp. 837-840, 1948 (Russian).

[2] W. A. Kirk, "A fixed point theorem for mappings which do not increase distances," The American Mathematical Monthly, vol. 72, pp. 1004-1006, 1965.

[3] K. Goebel and W. A. Kirk, Topics in Metric Fixed Point Theory, vol. 28 of Cambridge Studies in Advanced Mathematics, Cambridge University Press, Cambridge, UK, 1990.

[4] M. A. Khamsi and W. A. Kirk, "Pure and applied mathematics," in An Introduction to Metric Spaces and Fixed Point Theory, pp. 303-304, Wiley-Interscience, New York, NY, USA, 2001.

[5] A. Amini-Harandi, "Best proximity points theorems for cyclic strongly quasi-contraction mappings," Journal of Global Optimization, vol. 56, no. 4, pp. 1667-1674, 2012.

[6] W. A. Kirk, P. S. Srinivasan, and P. Veeramani, "Fixed points for mappings satisfying cyclical contractive conditions," Fixed Point Theory, vol. 4, no. 1, pp. 79-89, 2003.

[7] A. A. Eldred, W. A. Kirk, and P. Veeramani, "Proximal normal structure and relatively nonexpansive mappings," Studia Mathematica, vol. 171, no. 3, pp. 283-293, 2005.

[8] A. A. Eldred and P. Veeramani, "Existence and convergence of best proximity points," Journal of Mathematical Analysis and Applications, vol. 323, no. 2, pp. 1001-1006, 2006.

[9] A. Abkar and M. Gabeleh, "Best proximity points for asymptotic cyclic contraction mappings," Nonlinear Analysis: Theory, Methods \& Applications, vol. 74, no. 18, pp. 7261-7268, 2011.

[10] M. A. Al-Thagafi and N. Shahzad, "Convergence and existence results for best proximity points," Nonlinear Analysis: Theory, Methods \& Applications, vol. 70, no. 10, pp. 3665-3671, 2009.

[11] C. Di Bari, T. Suzuki, and C. Vetro, "Best proximity points for cyclic Meir-Keeler contractions," Nonlinear Analysis: Theory, Methods \& Applications, vol. 69, no. 11, pp. 3790-3794, 2008.

[12] R. Espínola, "A new approach to relatively nonexpansive mappings," Proceedings of the American Mathematical Society, vol. 136, no. 6, pp. 1987-1995, 2008.

[13] A. Fernández-León, "Existence and uniqueness of best proximity points in geodesic metric spaces," Nonlinear Analysis: Theory, Methods \& Applications, vol. 73, no. 4, pp. 915-921, 2010.

[14] M. Gabeleh, "Best proximity points for weak proximal contractions," Malaysian Mathematical Sciences Society, In press.

[15] M. Gabeleh, "Proximal weakly contractive and proximal nonexpansive non-self-mappings in metric and Banach spaces," Journal of Optimization Theory and Applications, vol. 158, no. 2, pp. 615-625, 2013.

[16] M. Gabeleh, "Best proximity points: global minimization of multivalued non-self mappings," Optimization Letters, 2013.

[17] C. Mongkolkeha and P. Kumam, "Best proximity point theorems for generalized cyclic contractions in ordered metric spaces," Journal of Optimization Theory and Applications, vol. 155, no. 1, pp. 215-226, 2012.

[18] B. Piątek, "On cyclic Meir-Keeler contractions in metric spaces," Nonlinear Analysis: Theory, Methods \& Applications, vol. 74, no. 1, pp. 35-40, 2011.

[19] S. Rezapour, M. Derafshpour, and N. Shahzad, "Best proximity points of cyclic $\phi$-contractions on reflexive Banach spaces," Fixed Point Theory and Applications, vol. 2010, Article ID 946178, 7 pages, 2010. 
[20] S. S. Basha and N. Shahzad, "Best proximity point theorems for generalized proximal contractions," Fixed Point Theory and Applications, vol. 2012, article 42, 2012.

[21] S. Sadiq Basha, N. Shahzad, and R. Jeyaraj, "Best proximity points: approximation and optimization," Optimization Letters, vol. 7, no. 1, pp. 145-155, 2013.

[22] S. S. Basha, N. Shahzad, and R. Jeyaraj, "Best proximity point theorems for reckoning optimal approximate solutions," Fixed Point Theory and Applications, vol. 2012, article 202, 2012.

[23] M. A. Al-Thagafi and N. Shahzad, "Best proximity pairs and equilibrium pairs for Kakutani multimaps," Nonlinear Analysis: Theory, Methods \& Applications, vol. 70, no. 3, pp. 1209-1216, 2009.

[24] M. A. Al-Thagafi and N. Shahzad, "Best proximity sets and equilibrium pairs for a finite family of multimaps," Fixed Point Theory and Applications, vol. 2008, Article ID 457069, 10 pages, 2008.

[25] A. Abkar and M. Gabeleh, "Proximal quasi-normal structure and a best proximity point theorem," Journal of Nonlinear and Convex Analysis, vol. 14, pp. 653-659, 2013.

[26] T. C. Lim, "A fixed point theorem for families on nonexpansive mappings," Pacific Journal of Mathematics, vol. 53, pp. 487-493, 1974.

[27] G. S. R. Kosuru and P. Veeramani, "A note on existence and convergence of best proximity points for pointwise cyclic contractions," Numerical Functional Analysis and Optimization, vol. 32, no. 7, pp. 821-830, 2011. 


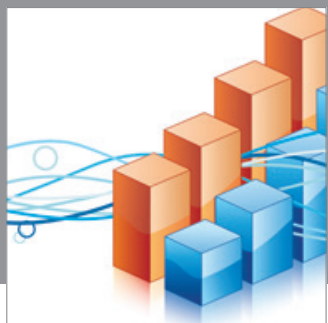

Advances in

Operations Research

mansans

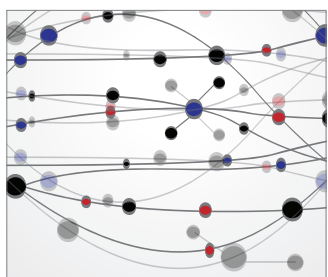

The Scientific World Journal
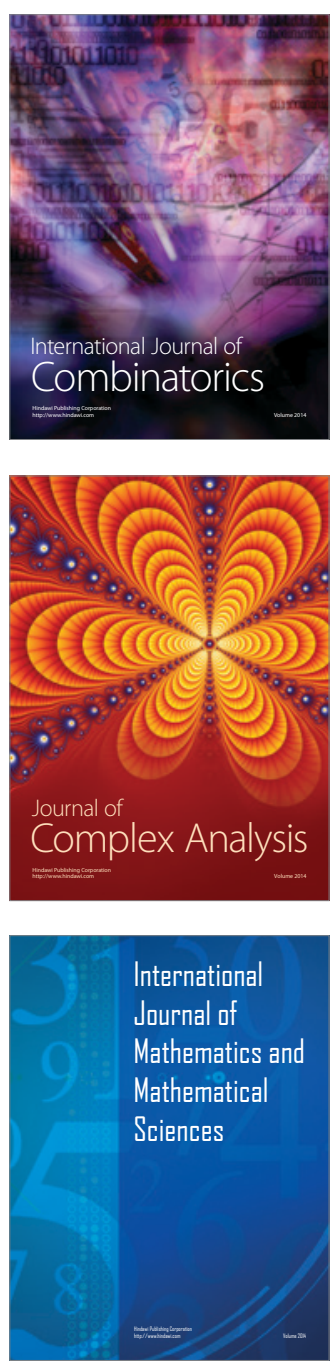
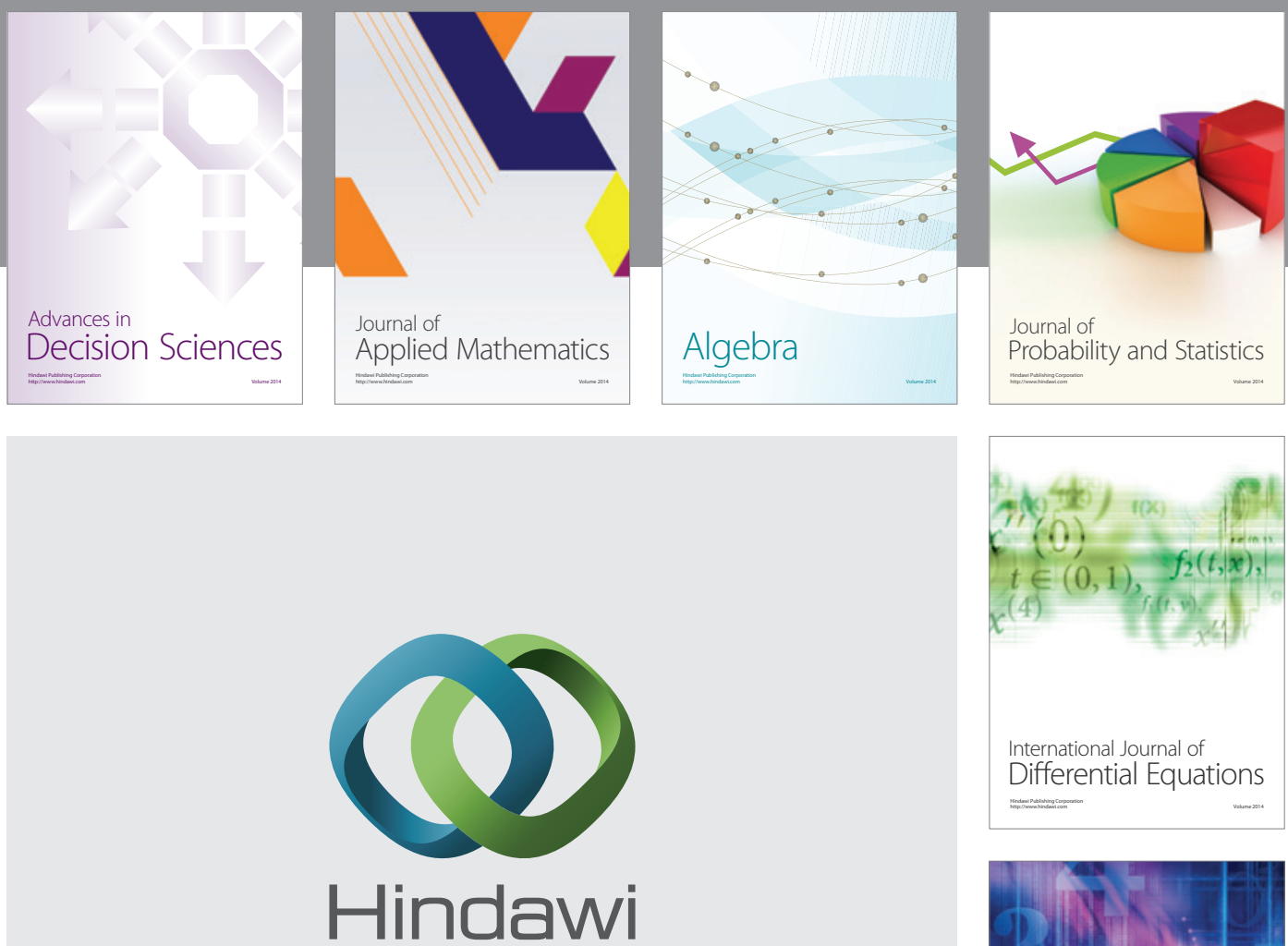

Submit your manuscripts at http://www.hindawi.com
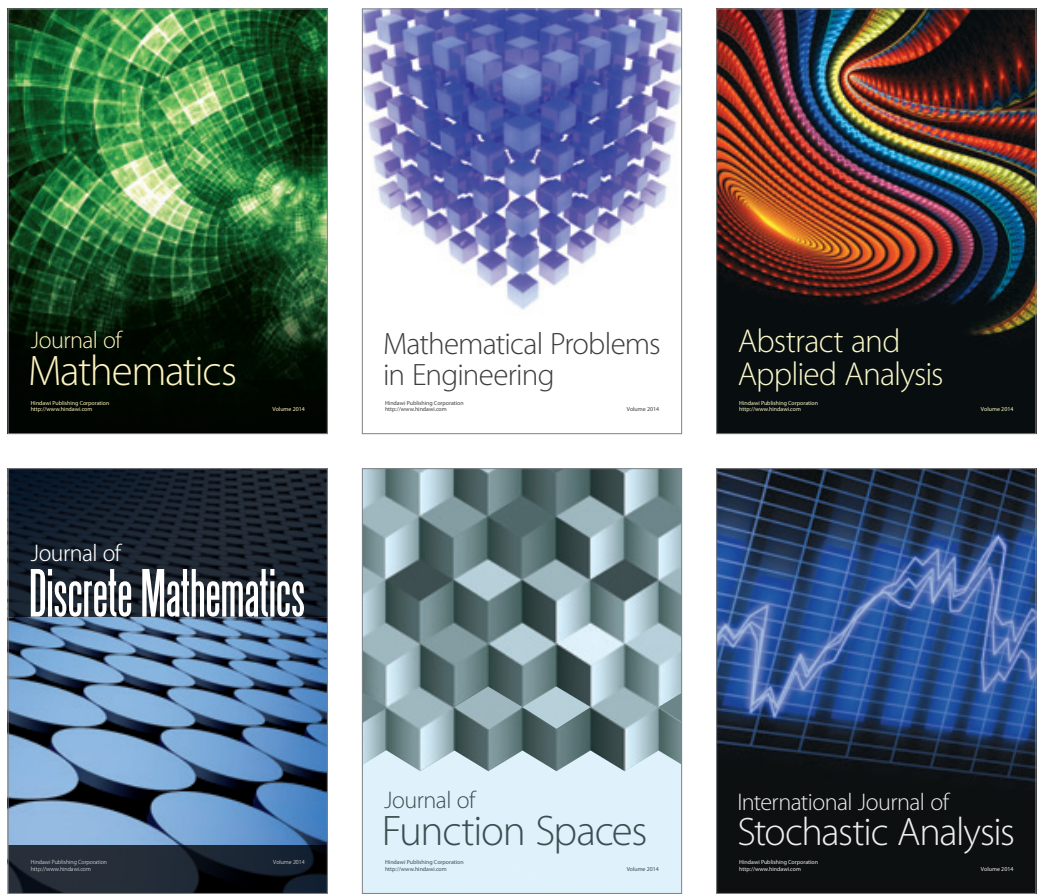

Journal of

Function Spaces

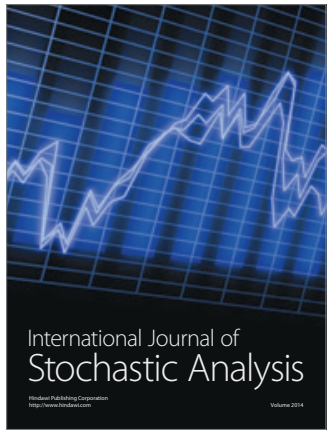

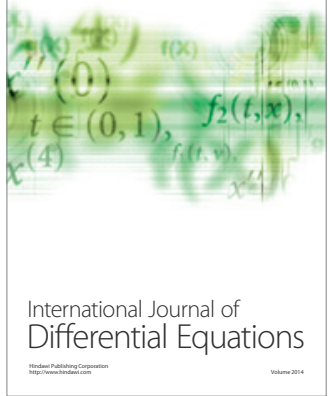
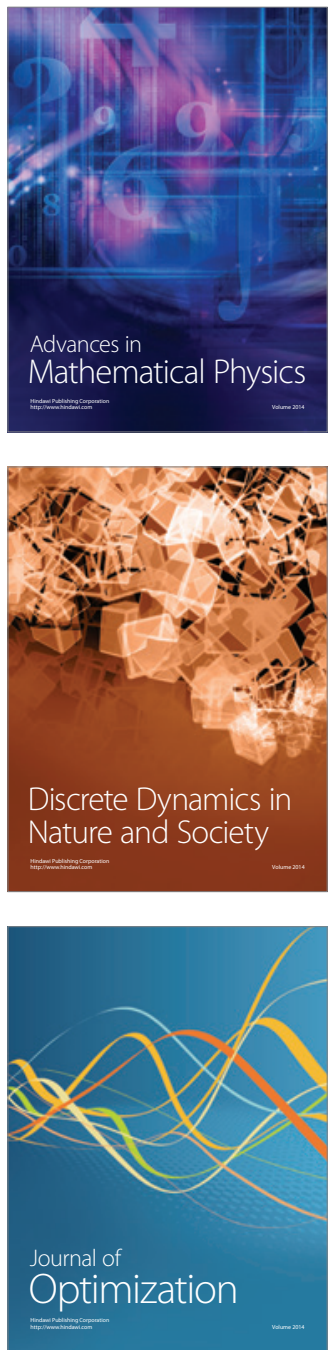